\title{
Exacerbation of disordered eating behaviors in adolescents with type 1 diabetes during the COVID-19 pandemic
}

\author{
Michal Gillon-Keren ${ }^{1,2}$ (D) Tamar Propper-Lewinsohn ${ }^{1} \cdot$ Maayan David $^{1} \cdot$ Alon Liberman $^{1} \cdot$ Moshe Phillip $^{1,3} \cdot$ \\ Tal Oron ${ }^{1,3}$
}

Received: 31 December 2021 / Accepted: 7 February 2022 / Published online: 7 March 2022

(c) Springer-Verlag Italia S.r.l., part of Springer Nature 2022

Keywords COVID-19 . Type 1 diabetes $\cdot$ Disordered eating behaviors $\cdot$ Eating habits $\cdot$ Eating disorders $\cdot$ Adolescent

\section{Introduction}

Nutritional therapy is essential for achieving proper metabolic control in type 1 diabetes (T1D) treatment. In our center, nutritional education endorses the Mediterranean diet known to prevent diabetes-related complications and improve overall health. Nevertheless, the continuous attention given to dietary restrictions can lead to disordered eating behaviors (DEB) in adolescents with T1D, which are associated with poor glycemic control and long-term complications [1]. The COVID-19 pandemic, especially during lockdowns, has a profound emotional impact on adolescents. With the disrupted school routine, reduced extracurricular activities, and increased use of social media, adolescents are more vulnerable to developing DEB [2].

This study examines whether DEB was exacerbated in adolescents with T1D during the COVID-19 pandemic and whether changes in glycemic control and dietary habits related to the Mediterranean diet occurred.

Managed by Antonio Secchi.

Michal Gillon-Keren and Tamar Propper-Lewinsohn should be considered a joint first author.

Michal Gillon-Keren

michalgk@gmail.com

1 The Institute of Endocrinology and Diabetes, Schneider Children's Medical Center, Petah Tikva 4920235, Israel

2 Faculty of Sciences, Kibbutzim College of Education Technology and the Arts, Tel Aviv 6250769, Israel

3 Sackler Faculty of Medicine, Tel Aviv University, Tel Aviv 6997801, Israel

\section{Methods}

This is an observational, longitudinal study evaluating the dietary habits of adolescents aged $15-19$ years with T1D for more than one year, treated at the diabetes clinic in Schneider Children's Medical Center of Israel. As a routine in our clinic, adolescents aged 15-19 complete a DEB screening tool and a nutritional questionnaire. The Diabetes Eating Problem Survey-Revised (DEPS-R) is used to assess the risk of DEB (range 0-80; score $>20$ indicates a high risk for DEB) [3] and the Mediterranean Diet Quality Index (KIDMED) questionnaire for children and adolescents to assess the diet quality according to the Mediterranean diet pattern (range -4 to 16 points) [4].

The participants' dietary habits before the COVID-19 pandemic (February 2019 to January 2020) and near the end of the first lockdown (May to June 2020) were studied. The study participants were recruited near the end of the first lockdown, where they completed the questionnaires for the second time. Exclusion criteria were medical conditions related to nutritional status, psychiatric disorders, or inability to complete the study questionnaires.

Clinical data, including age, gender, weight, height, and glycated hemoglobin (HbA1c), were retrieved from the patient's medical record at baseline and re-measured at the first visit after the lockdown. Body mass index (BMI) was calculated as weight $(\mathrm{kg}) / \mathrm{height}^{2}\left(\mathrm{~m}^{2}\right)$ and standardized to age and gender. BMI above the 85th percentile for age and gender was classified as overweight or obese, and below the 5th percentile as underweight. Capillary $\mathrm{HbA} 1 \mathrm{c}$ was measured by the DCA 2000 analyzer (Bayer Diagnostics Inc). The Rabin Medical Center ethics committee approved the study. Participants or their legal guardians provided informed consent approving their participation. 


\section{Statistical analyses}

IBM SPSS Statistics version 21.0 (Armonk, NY: IBM Corp.) was used for analysis. The mean differences between the questionnaire scores were analyzed using paired t-tests. The associations between the questionnaires scores and potential explanatory variables were analyzed using independent t-tests or Pearson's correlations. Multiple linear regression analysis was performed to examine the association between the changes in DEPS-R scores and potential explanatory variables. An alpha level of 0.05 was used for all statistical analyses.

\section{Results}

Thirty-four adolescents (mean age $17.1 \pm 1.1$ years; $38 \%$ females) were included in the study. The average time between baseline and follow-up was $11.5+4.3$ ) months.

DEPS-R score significantly increased during the COVID19 lockdown $(12.7 \pm 7.4$ vs. $16.0 \pm 10.0, p=0.01)$ (Table 1$)$. Eleven participants had a score $>20$ points, indicating a high risk for DEB, compared to six participants at baseline $(p=0.18)$. No significant correlations were found between the changes in DEPS-R score and age, gender, HbA1c, or BMI. There was no change in KIDMED scores, and no correlations were found between changes in KIDMED scores and age, gender, $\mathrm{HbA1c}$, or BMI.

\section{Discussion}

This study examined changes in DEB and dietary habits in adolescents with T1D during the COVID-19 pandemic. Our results suggest that the COVID-19 lockdown negatively affected the eating behaviors of adolescents with T1D, doubling the probability of having DEB. The pandemic did not affect glycemic control, BMI, or adherence to the Mediterranean diet.

The COVID-19 pandemic has been shown to increase the incidence of eating disorders among adolescents [5]. Our results suggest that the COVID-19 exacerbated DEB in adolescents with T1D.

Several factors such as lockdowns, restrictions, and altered sleeping patterns can explain the increase in DEB. Also, social isolation and intensive media and social media exposure may increase stress and anxiety, which adversely affect eating habits [2].

In our study, the drive for thinness and changes in meal patterns were the main factors aggravating DEB. Although no significant alterations in BMI were observed, more than a quarter of the participants perceived themselves as gaining weight during the pandemic. The changes in meal patterns can be related to the fear of the disease and its complications, motivating the parents to be more careful about their children's diet on the one hand. On the other hand, the increased parental presence may have caused more stress and a desire to eat alone. Glycemic control and dietary patterns did not change significantly in our patients. Thus, we believe that the worsening in DEB is not related to diabetes management but to eating pathology and the drive for thinness. The main limitation of our study is its small sample size. However, the study's design enabled us to dissect the pandemic's effect on the participants' eating behaviors as the baseline questionnaires were completed before the pandemic. Nevertheless, further studies on larger populations are needed to confirm our findings.

In conclusion, our study shows an exacerbation in DEB among adolescents with T1D during the COVID-19 outbreak. The exacerbation is likely due to eating pathology and the drive for thinness and not because of poor diabetes management. As the COVID-19 pandemic is far from over, our study emphasizes the importance of routine screenings for DEB in adolescents with type 1 diabetes.
Table 1 Participant characteristics at baseline and near the end of the first lockdown

\begin{tabular}{lllrl}
\hline Characteristic & Baseline $($ Mean \pm SD) & COVID-19 $($ Mean \pm SD) & T & $p$-value \\
\hline BMI z-score & $0.1 \pm 1.0$ & $0.1 \pm 0.9$ & 0.78 & 0.44 \\
HbA1c \% & $7.9(7.3-8.8)$ & $7.9(7.3-8.9)$ & -0.53 & 0.60 \\
mmol/mol* $_{\text {KIDMED score** }}^{* *}$ & $63(56-73)$ & $63(56-74)$ & & \\
DEPS-R score*** & $12.7 \pm 7.7$ & $4.8 \pm 2.4$ & 0.75 & 0.46 \\
\hline
\end{tabular}

*Data presented as median (interquartile range)

$* *$ Score range (-4)-16; score $\leq 3$ indicates poor adherence, and score $\geq 8$ indicates good adherence to the Mediterranean diet

*** Score range $0-80 ;$ score $\geq 20$ indicates a high risk for DEB

$\mathrm{SD}$, standard deviation; BMI, body mass index; HbAlc, glycated hemoglobin; KIDMED, Mediterranean Diet Quality Index in children and adolescents; DEPS-R, Diabetes-specific Eating Problems SurveyRevised 
Acknowledgements The authors thank Gila Fayman, Fatima Essa, and Idit Vardi for their help in data collection, as well as the patients and their parents who agreed to participate in the study.

Author contributions MGK, TPL, AL, and MP were involved in conceptualization; MGK, TPL, and MP contributed to methodology; MD, MGK, and TPL collected the data; TPL and MGK carried out the formal analysis and investigation; MGK, TPL, and AL wrote and prepared the original draft; TO, MP, MGK, and TPL took part in writing, reviewing, and editing; and MGK, TPL, and TO participated in supervision.

Funding This research did not receive any specific grant from funding agencies in the public, commercial, or not-for-profit sectors.

Data availability The data that support the findings of this study are available from the corresponding author upon reasonable request.

\section{Declarations}

Conflict of interest The authors declare no conflict of interest.

Ethics approval The study received ethical approval from the Rabin Medical Center ethics committee.

Informed consent All participants or their legal guardians provided informed consent.

\section{References}

1. Nip ASY, Reboussin BA, Dabelea D et al (2019) Disordered eating behaviors in youth and young adults with type 1 or type
2 diabetes receiving insulin therapy: the search for diabetes in youth study. Diabetes Care 42:859-866. https://doi.org/10.2337/ dc $18-2420$

2. Rodgers RF, Lombardo C, Cerolini S et al (2020) The impact of the COVID-19 pandemic on eating disorder risk and symptoms. Int J Eat Disord 53:1166-1170. https://doi.org/10.1002/eat.23318

3. Markowitz JT, Butler DA, Volkening LK et al (2010) Brief screening tool for disordered eating in diabetes: internal consistency and external validity in a contemporary sample of pediatric patients with type 1 diabetes. Diabetes Care 33:495-500. https://doi.org/ $10.2337 / \mathrm{dc} 09-1890$

4. Serra-Majem L, Ribas L, Ngo J et al (2004) Food, youth and the Mediterranean diet in Spain. Development of KIDMED, Mediterranean Diet Quality Index in children and adolescents. Public Health Nutr 7:931-935. https://doi.org/10.1079/phn2004556

5. Agostino H, Burstein B, Moubayed D et al (2021) Trends in the Incidence of new-onset anorexia nervosa and atypical anorexia nervosa among youth during the COVID-19 pandemic in Canada. JAMA Netw Open 4:e2137395. https://doi.org/10.1001/jaman etworkopen.2021.37395

Publisher's Note Springer Nature remains neutral with regard to jurisdictional claims in published maps and institutional affiliations. 